\title{
Characterization of tau oligomeric seeds in progressive supranuclear palsy
}

\author{
Julia E Gerson ${ }^{1,2}$, Urmi Sengupta ${ }^{1,2}$, Cristian A Lasagna-Reeves ${ }^{1,2}$, Marcos J Guerrero-Muñoz ${ }^{1,2}$, Juan Troncoso ${ }^{3}$ \\ and Rakez Kayed ${ }^{1,2^{*}}$
}

\begin{abstract}
Background: Progressive supranuclear palsy (PSP) is a neurodegenerative tauopathy which is primarily defined by the deposition of tau into globose-type neurofibrillary tangles (NFT). Tau in its native form has important functions for microtubule dynamics. Tau undergoes alternative splicing in exons 2, 3, and 10 which results in six different isoforms. Products of splicing on exon 10 are the most prone to mutations. Three repeat (3R) and four repeat (4R) tau, like other disease-associated amyloids, can form oligomers which may then go on to further aggregate and form fibrils. Recent studies from our laboratory and others have provided evidence that tau oligomers, not NFTs, are the most toxic species in neurodegenerative tauopathies and seed the pathological spread of tau.

Results: Analysis of PSP brain sections revealed globose-type NFTs, as well as both phosphorylated and unphosphorylated tau oligomers. Analysis of PSP brains via Western blot and ELISA revealed the presence of increased levels of tau oligomers compared to age-matched control brains. Oligomers were immunoprecipitated from PSP brain and were capable of seeding the oligomerization of both $3 R$ and $4 R$ tau isoforms.

Conclusions: This is the first time tau oligomers have been characterized in PSP. These results indicate that tau oligomers are an important component of PSP pathology, along with NFTs. The ability of PSP brain-derived tau oligomers to seed $3 R$ and $4 R$ tau suggests that these oligomers represent the pathological species responsible for disease propagation and the presence of oligomers in a pure neurodegenerative tauopathy implies a common neuropathological process for tau seen in diseases with other amyloid proteins.
\end{abstract}

Keywords: Tau, Oligomers, Progressive supranuclear palsy, Seeding

\section{Introduction}

Progressive supranuclear palsy (PSP) is a common parkinsonian neurodegenerative tauopathy which is characterized by the development of dementia, changes to personality, visual and speech deficiencies, and gait alteration [1]. It affects about 6.5 in 100,000 people [2]. Pathologically, PSP is primarily defined by the deposition of tau into neurofibrillary tangles (NFT). Tau in its native form has important functions for microtubule stabilization and neurite growth. Tau undergoes alternative splicing in exons 2, 3, and 10 which results in six different isoforms of tau protein in the adult central nervous system [3]. Products of splicing on

\footnotetext{
* Correspondence: rakayed@utmb.edu

${ }^{1}$ Mitchell Center for Neurodegenerative Diseases, University of Texas Medical Branch, Galveston, TX 77555, USA

${ }^{2}$ Departments of Neurology, Neuroscience and Cell Biology, University of

Texas Medical Branch, Galveston, TX 77555, USA

Full list of author information is available at the end of the article
}

exon 10 are the most prone to mutations and result in three isoforms with three microtubule binding repeats (3R tau) and three isoforms with four microtubule binding repeats ( $4 \mathrm{R}$ tau). As the sequence corresponding to exon 10 appears to be of great importance in increasing the affinity of tau for microtubules [4], differences between $3 R$ and $4 R$ tau may have implications for microtubule dynamics, as well as for localization of tau to the microtubules. This is of great importance because tau with low affinity for microtubules may be released into the cytosol, where it is free to form aggregates. It is unknown whether the seeding potential of oligomers from $4 \mathrm{R}$ tau differs from that of $3 R$ tau and whether this may underlie some of the differences between various tauopathies specific to the number of microtubule-binding repeats.

PSP, unlike tauopathies such as Alzheimer's disease (AD) which form aggregates from both tau forms, has a shifted ratio of 4R:3R tau [5] and specifically forms NFTs 
from $4 \mathrm{R}$ tau [6]. Similarly, corticobasal degeneration (CBD) also exhibits aggregates from 4R tau alone [6]. However, tau isolated from CBD and PSP display different fragmentation due to variation in cleavage at the amino terminal, suggesting that the two diseases have different mechanisms of proteolytic processing for tau aggregates $[7,8]$. Additionally, though most of the phosphorylation sites of misfolded tau in PSP are similar to the other tauopathies, there are some differences $[9,10]$. These differences may underlie the unique pathological features and spatial organization of tau aggregation between each disease, which likely lead to differences in functional outcome. The two main pathological hallmarks of PSP include globose-type NFTs and tuft-shaped astrocytes, while other neurodegenerative tauopathies display different histological tau features $[1,11,12]$. The presence of tau aggregation in glial cells is a prominent feature in PSP. In addition to the presence of tufted astrocytes, oligodendrocytes may be affected in PSP as well. Argophylic threads and coiled bodies comprised of $4 \mathrm{R}$ tau have been found in oligodendrocytes in PSP cases [13]. Tau pathology is also located in different regions in PSP than in other diseases. NFTs in PSP are seen in the brainstem, basal ganglia, and the prefrontal and precentral cortex and hippocampus [12,14]. Therefore, while tauopathies do share many common features, it is important to study the mechanism of each individually.

While NFTs are the main histological hallmark in PSP, tau, like other disease-associated amyloids, can form oligomers which then go on to form fibrils. Recent studies from our laboratory and others have shown that tau oligomers, not NFTs, are the most toxic species in vitro and in vivo and may seed the pathological spread of tau [15-18]. Tau oligomers have been shown to be present in the brains of patients with Alzheimer's disease before NFTs can be detected [16,19-21], correlating with dysfunction of the ubiquitin proteasome system and mitochondria [22,23]. Moreover, cognitive and motor deficits in animal models of tauopathies correspond to levels of tau oligomers, but not to levels of NFTs [15,24-28]. However, the role of oligomeric tau has never been investigated in PSP, though the importance of oligomeric tau in the spread of pathology in PSP has been suggested [29]. In this study, we characterized tau oligomers for the first time in human PSP brain samples and showed the potential for them to seed the oligomerization of both $3 \mathrm{R}$ and $4 \mathrm{R}$ tau, implicating oligomeric tau as an important component of PSP disease progression.

\section{Materials and methods}

\section{Preparation of brain homogenate}

Frozen brain tissue from the pons was attained from subjects with progressive supranuclear palsy (PSP) and age-matched control subjects from the Institute for
Brain Aging and Dementia (University of CaliforniaIrvine, Irvine, California, USA) and the Brain Resource Center at Johns Hopkins. All postmortem brain tissue used were randomized. PSP tissue cases were collected with patient consent and handled under protocols approved by the Johns Hopkins Institutional Review Board. PSP samples were examined at the Division of Neuropathology at John Hopkins University. Brains were homogenized in PBS with a protease inhibitor cocktail (catalog no. 11836145001; Roche Applied Science, Indianapolis, IN, USA), using a 1:3 dilution of brain: PBS $(\mathrm{w} / \mathrm{v})$. Samples were centrifuged at $10000 \mathrm{rpm}$ for 10 min at $4^{\circ} \mathrm{C}$. Supernatants were aliquoted, snap-frozen, and stored at $-80^{\circ} \mathrm{C}$ until use.

\section{Immunofluorescence}

Sections used for fluorescent immunohistochemistry were deparaffinized, rehydrated, and blocked in normal goat serum for $1 \mathrm{hr}$ and incubated overnight with antitau oligomer-specific polyclonal antibody, T22 (1:300). The next day, sections were washed three times for 10 min in PBS and incubated with goat anti-rabbit IgG Alexa-568 (1:500; Invitrogen) for $1 \mathrm{hr}$. Sections were again washed three times for 10 minutes in PBS and were either incubated overnight with Tau-5 (1:300) for total tau, AT8 (1:100) for Ser202/Thr205 phosphorylated tau, or PHF13 (1:250) for Ser396 phosphorylated tau. The following day, sections were washed in PBS three times for 10 minutes, then incubated with donkey antimouse IgG Alexa-Fluor 488 (1:500; Invitrogen). Sections were washed and incubated in DAPI (Invitrogen), then mounted using Fluoromount G (Southern Biotech) mounting medium. Sections were imaged using a Zeiss LSM 510 Meta confocal system. Six images were taken from each sample and cells were randomly selected from each image for quantification using Image-J. The total level of fluorescence was measured for each cell, as well as the level of background from three different regions around the cell without fluorescence. In order to correct the level of fluorescence for background and cell size, the background multiplied by the area of the cell was subtracted from the total fluorescence. The corrected cell fluorescence was analyzed via One-way Analysis of Variance (ANOVA).

\section{Western blot}

Pre-cast NuPAGE 4-12\% Bis-Tris Gels for SDS-PAGE (Invitrogen) were loaded with 20-25 $\mu \mathrm{g}$ of protein for each sample per well, run under reducing conditions, and then transferred to nitrocellulose membranes. Membranes were then blocked overnight at $4^{\circ} \mathrm{C}$ with $10 \%$ nonfat dried milk. The next day membranes were incubated with T22 (1:250) for tau oligomers, Tau-5 (1:1000) for total tau, and GAPDH (1:1000; Sigma) as a loading control, diluted in 5\% nonfat dried milk for $1 \mathrm{hr}$ at room 
temperature. Tau-5 and GAPDH immunoreactivity were detected with horseradish peroxidase-conjugated IgG anti-mouse secondary antibody (1:3000, GE Healthcare) and T22 was detected with horseradish peroxidaseconjugated IgG anti-rabbit secondary antibody (1:3000, GE Healthcare). For signal detection, ECL plus (GE Healthcare) was used. Densitometry of each band was quantified and normalized with GAPDH using Image-J and analyzed by one-way ANOVA.

\section{ELISA}

For ELISA analysis, 96-well plates were coated with $15 \mu \mathrm{l}$ of samples (PBS soluble fractions of brains) using $0.05 \mathrm{M}$ sodium bicarbonate $(\mathrm{pH} 9.6)$ as the coating buffer and incubated overnight at $4{ }^{\circ} \mathrm{C}$. Plates were washed once with TBST (0.01\% Tween 20), then blocked for $2 \mathrm{hrs}$ at RT with $10 \%$ non-fat milk. Plates were then washed once with TBST. T22 (1:250) or Tau-5 (1:1000) diluted in $5 \%$ nonfat milk was added and allowed to react for 1 $\mathrm{hr}$ at RT. Plates were washed three times with TBST. T22 and Tau-5 immunoreactivity was detected using $100 \mu \mathrm{l}$ of HRP-conjugated anti-rabbit IgG (GE Healthcare) or $100 \mu \mathrm{l}$ of HRP-conjugated anti-mouse IgG (GE Healthcare) respectively diluted 1:3000 in 5\% nonfat milk and incubated for $1 \mathrm{hr}$ at RT. Lastly, plates were washed three times with TBST and incubated with 100ul of 3,3,5,5-tetramethylbenzidine (TMB-1 component substrate, from Dako) for $1 \mathrm{hr}$ in the dark. To stop the reaction, $100 \mu \mathrm{l} 2 \mathrm{M} \mathrm{HCl}$ was applied and plates were read at $450 \mathrm{~nm}$ in a Polar Star Omega plate reader (BMG Labtech). Each sample was measured in triplicate and results were analyzed by student's t-test.

Preparation of PSP brain-derived tau oligomers (BDTO)

The immunoprecipitation of tau oligomers from PSP pons was completed as previously described $[17,30]$. Thirty $\mu \mathrm{L}$

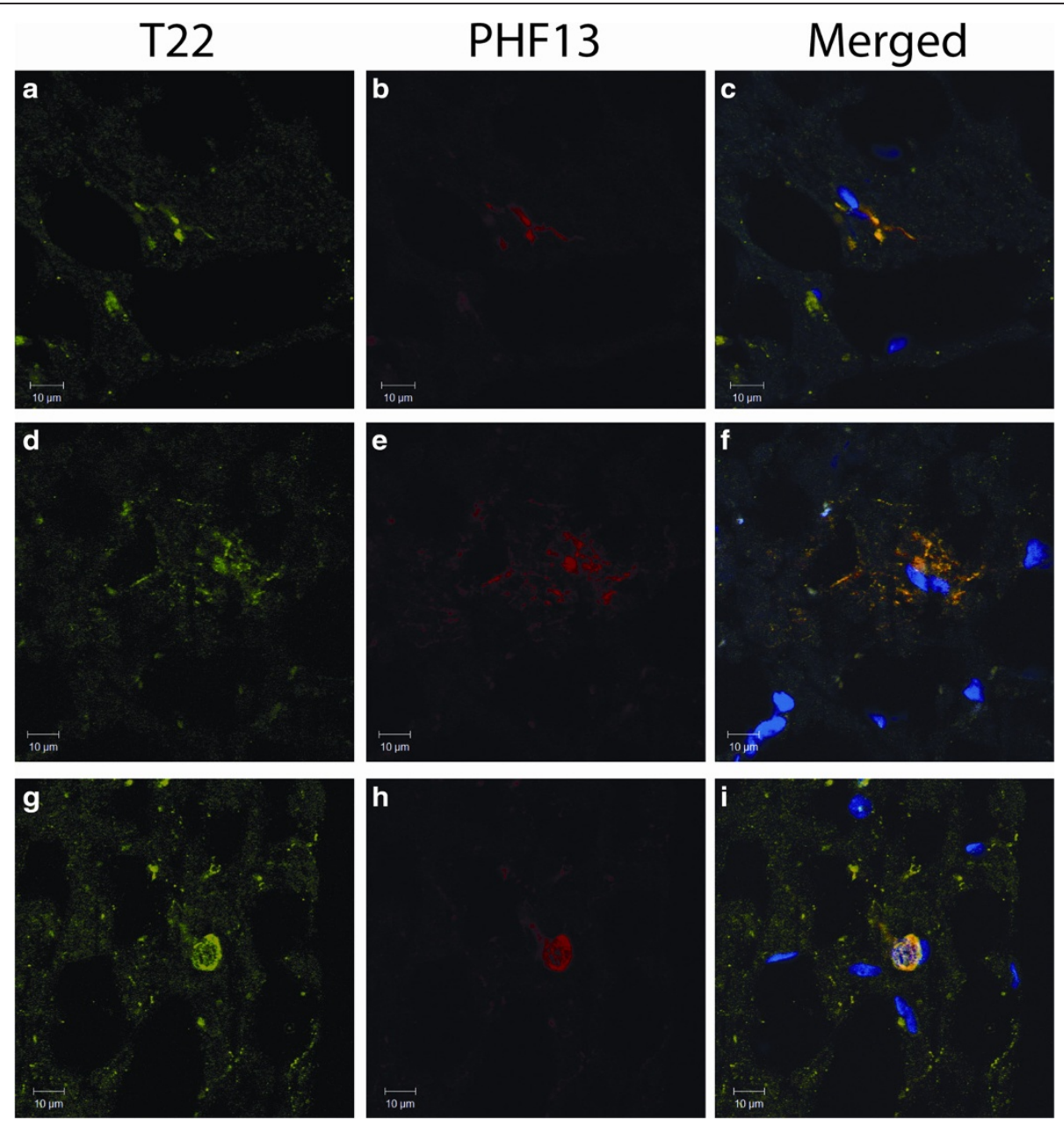

Figure 1 PSP brain sections labeled with T22 and PHF13 display characteristic PSP hallmarks. PSP brain sections immunofluorescently labeled with T22 (red) and PHF13 (green). Intracellular tau oligomers detected with T22 partially colocalize with PHF13 (a-c). Oligomers resembling tufted astrocytes or extracellular deposits were also detected to partially colocalize with phosphorylated tau recognized by PHF13 (d-f). Characteristic globose-type NFTs were detected by T22 and PHF13 (g-i). 
of tosyl-activated magnetic Dynabeads (Dynal Biotech, Lafayette Hill, PA) were coated with $20 \mu \mathrm{g}$ of anti-tau oligomer-specific polyclonal antibody, T22 $(1.0 \mathrm{mg} / \mathrm{ml}) \mathrm{di}-$ luted in $50 \mu \mathrm{L}$ of $0.1 \mathrm{M}$ borate, $\mathrm{pH} 9.5$, keeping end concentration of beads at $20 \mathrm{mg} / \mathrm{mL}$ overnight at $37^{\circ} \mathrm{C}$. Beads were washed $(0.2 \mathrm{M}$ Tris- $\mathrm{HCl}, 0.1 \%$ bovine serum albumin, $\mathrm{pH}$ 8.5) and then incubated with $100 \mu \mathrm{l}$ of PSP brain homogenate (PBS soluble fraction) with rotation at room temperature for $1 \mathrm{hr}$. Beads were washed three times with PBS and eluted using $0.1 \mathrm{M}$ glycine, $\mathrm{pH}$ 2.8. The $\mathrm{pH}$ was adjusted using $1 \mathrm{M}$ Tris- $\mathrm{HCl} \mathrm{pH} 8.0$ and then fractions were centrifuged in a microcon centrifugal filter device with a molecular weight cut-off of $25 \mathrm{kDa}$ (Millipore, Cat \# $42415)$ at $14000 \mathrm{~g}$ for $25 \mathrm{~min}$ at $4^{\circ} \mathrm{C}$. Oligomers were resuspended in sterile PBS. Protein concentration was measured using the bicinchoninic acid protein assay (Pierce). The samples were then centrifuged again in a microcon centrifugal filter device with a cut-off of $25 \mathrm{kDa}$ at $14000 \mathrm{~g}$ for $25 \mathrm{~min}$ at $4^{\circ} \mathrm{C}$. Oligomers were characterized by various methods including size-exclusion chromatography (SEC) and atomic force microscopy (AFM) as previously described $[17,31]$ and stored at $-80^{\circ} \mathrm{C}$. Oligomers were re-suspended in PBS in order to obtain the desired concentration $(0.18-1.2 \mathrm{mg} / \mathrm{ml})$ and kept at $4^{\circ} \mathrm{C}$ for $15-30$ $\mathrm{min}$, then at room temperature for $10 \mathrm{~min}$ before use. Oligomers were characterized as previously described [31].

\section{Seeding assay}

$3 R$ and $4 R$ tau monomer were obtained by dissolving lyophilized pellets of recombinant $3 R$ and $4 R$ tau at $0.3 \mathrm{mg} /$ $\mathrm{mL}$ concentration in phosphate-buffered saline (PBS) $[15,31]$ and seeded with PSP brain-derived tau oligomers isolated as described [17]. BDTO seeds were added at $1: 100(\mathrm{w} / \mathrm{w}$ ) to $2.5 \mu \mathrm{M}$ solutions of monomeric $3 \mathrm{R}$ and $4 \mathrm{R}$ tau in PBS (in duplicates) with gentle agitation at room temperature. Aliquots were taken at each time point and immediately added to ELISA plate wells containing coating buffer $(0.05 \mathrm{M}$ sodium bicarbonate buffer, $\mathrm{pH}$ 9.6, $0.02 \%$ sodium azide). Other aliquots were added to mica sheets for AFM analysis. Samples were analyzed by AFM and ELISA using T22 and Tau-5.

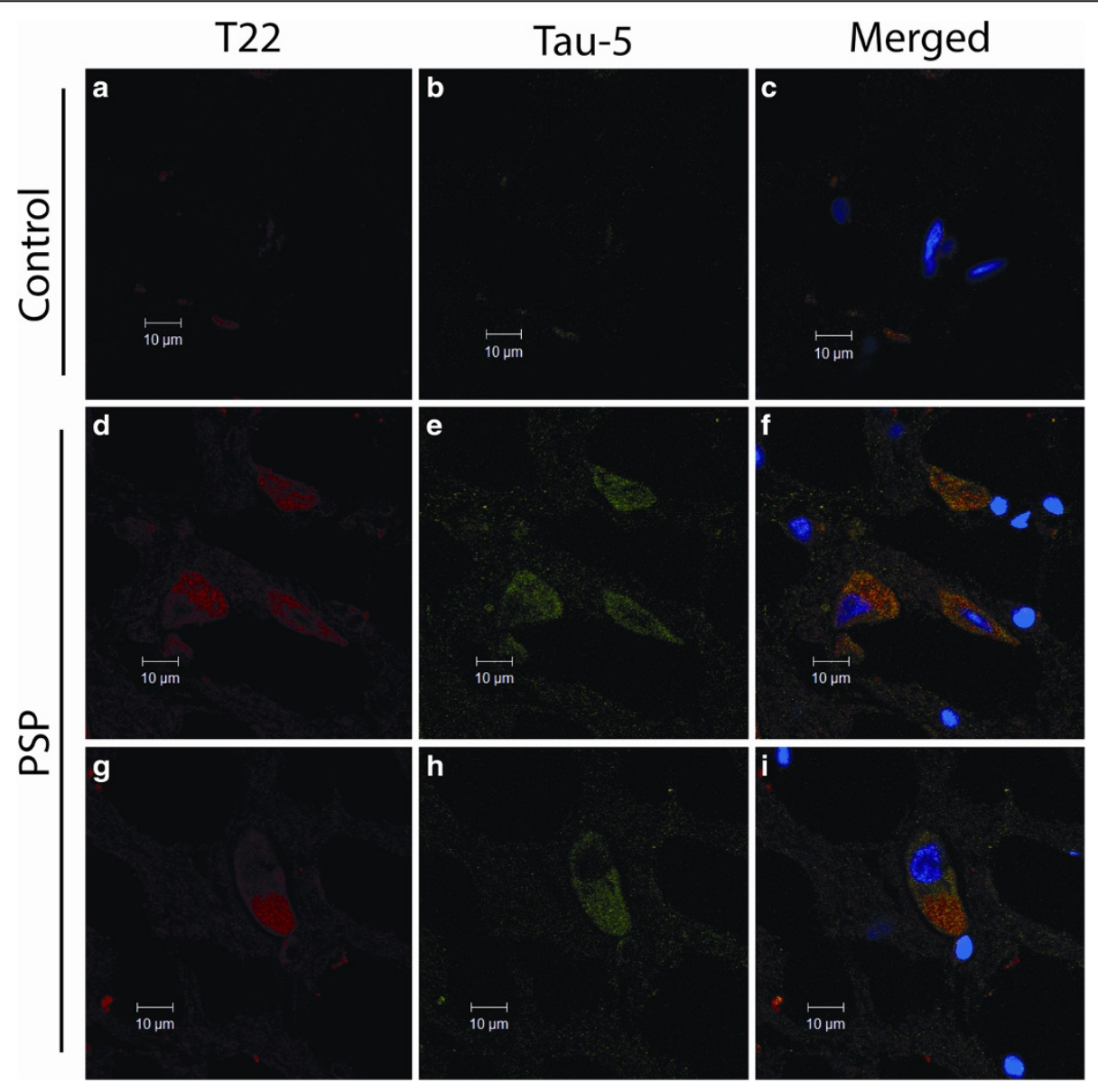

Figure 2 PSP brain sections express heightened levels of tau oligomers and total tau. PSP and age-matched control brain sections immunofluorescently labeled with T22 (red) and Tau-5 (green). Control brains do not exhibit NFTs labeled with Tau-5 or oligomeric tau (a-c). Globose-type NFTs are detected with Tau-5 and partially colocalize with tau oligomers (d-i). 


\section{Results}

Brain sections present with tau oligomers and classical PSP histological hallmarks

Sections of pons from PSP patients were labeled with T22, which is specific for tau oligomers, and with PHF13 which recognizes phosphorylated tau (Figure 1a-i). T22 was partially colocalized with PHF13 (Figure 1c, f, i), signifying the presence of both phosphorylated and unphosphorylated tau oligomers. Both extracellular and intracellular tau oligomers were recognized by T22. Tau accumulation resembling tufted astrocytes was observed to be co-labeled by T22 and PHF13 (Figure 1d-f). Globoseshaped NFTs comprised of phosphorylated tau and tau oligomers were also present (Figure 1g-i).

Tau oligomer and NFT pathology is present in PSP brains but not in age-matched controls

Pons sections from PSP patients and age-matched controls were labeled with T22 and Tau-5 for total tau. Control brains exhibited small punctate tau staining (Figure 2a-c), while PSP brains displayed an increased amount of both tau oligomers and total tau, as well as the presence of large, globose NFTs surrounding many of the cells (Figure 2d-i). T22 showed some overlap with Tau-5, as well as some distinct oligomeric tau foci. Staining with AT8, which recognizes phosphorylated tau commonly found in NFTs, and T22 did not exhibit reactivity with control brain sections (Figure 3a-c), but showed increased levels of tau oligomers in PSP brain, as well as NFTs and pre-NFTs positive for AT8 (Figure 3d-i). T22 staining was largely colocalized with AT8, signifying the presence of phosphorylated tau oligomers (Figure $3 f$ \& i).

\section{Tau oligomer levels are significantly higher in PSP versus} control brains

Analysis of PSP brains via Western blot with T22 and Tau-5 revealed the presence of high molecular weight tau oligomers (Figure 4a). Additionally, analysis by direct ELISA showed a significant increase in levels of tau oligomers detected with T22 in PSP patient brains compared to control brains (Figure 4b). Immunofluorescent staining of individual cells with T22, Tau-5, and AT8 was

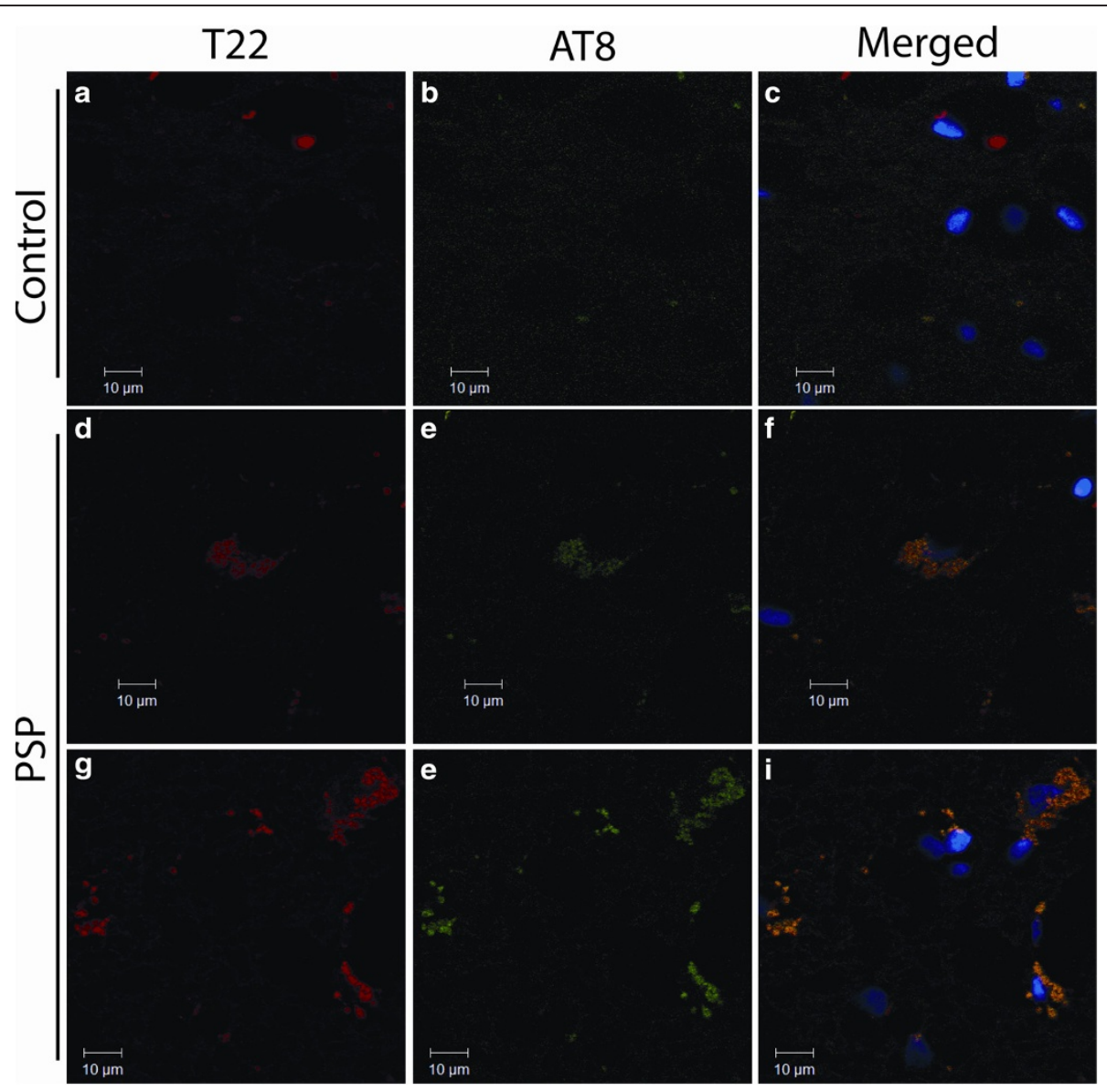

Figure 3 PSP brain sections display higher levels of tau oligomer and phosphorylated tau staining. PSP and age-matched control pons sections immunofluorescently labeled with T22 (red) and AT8 (green). Control brains do not exhibit phosphorylated tau or oligomeric tau deposition (a-c). Pre-NFTs and NFTs were detected with AT8, colocalizing with T22 (d-i). 


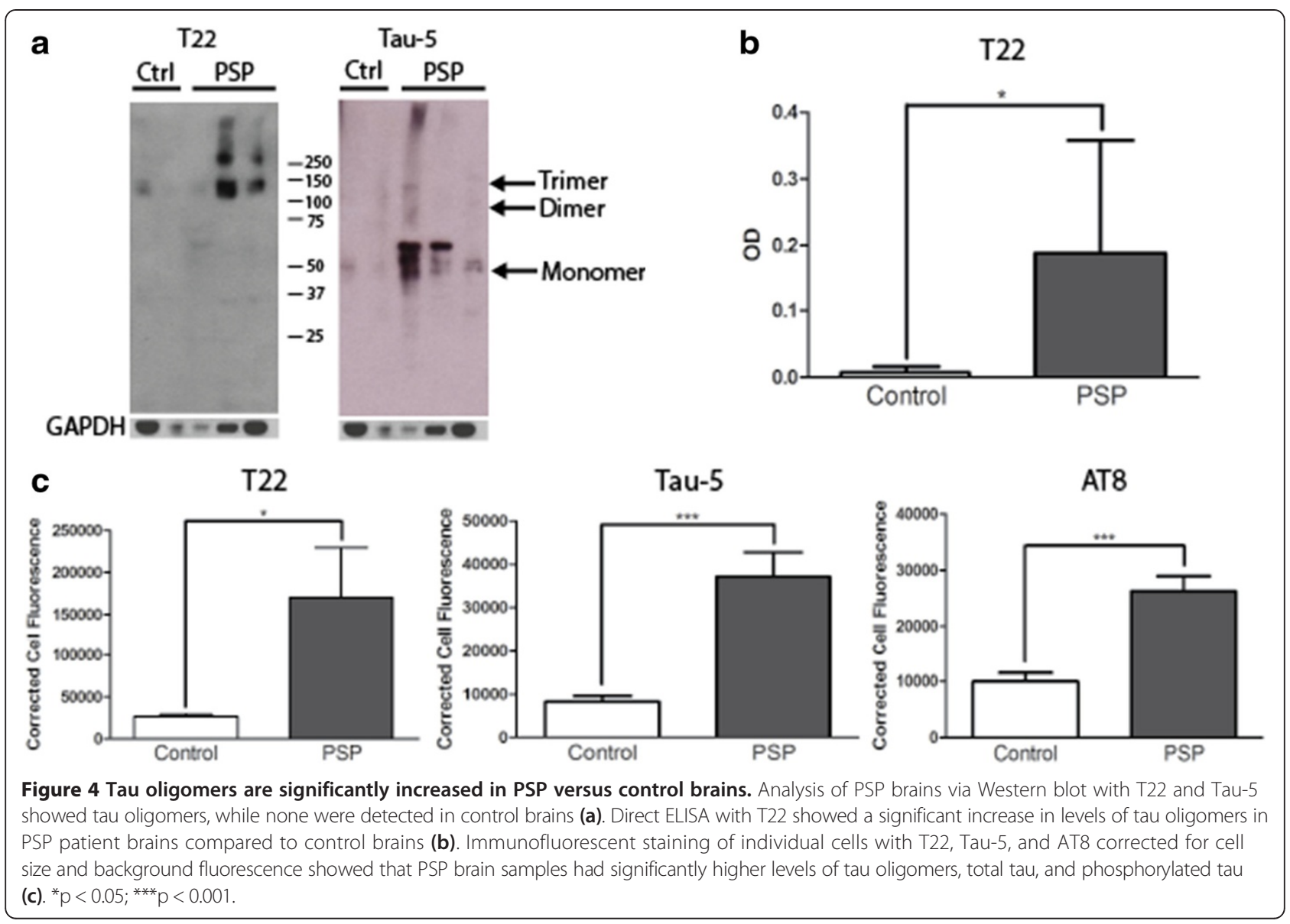

corrected for cell size and background fluorescence and quantified in PSP patients and controls. PSP brain samples had significantly higher levels of tau oligomers, total tau, and phosphorylated tau (Figure 4c).

\section{PSP brain-derived tau oligomers seed oligomerization of both $3 R$ and $4 R$ tau}

Tau oligomers were successfully immunoprecipitated from PSP brain, displaying characteristic oligomeric size and structure by analysis with AFM. Co-incubation of BDTO with $3 \mathrm{R}$ and $4 \mathrm{R}$ tau monomer induced the oligomerization of both isoforms of tau as shown by AFM (Figure 5a) and Western blot with Tau-5 (Figure 5b). Direct ELISA of 3R and $4 \mathrm{R}$ seeded with BDTO with Tau- 5 confirmed consistent concentrations of tau in all samples, while ELISA with T22 displays an increase in levels of tau oligomers as incubation time with oligomers increases (Figure 5c).

\section{Discussion}

Utilizing brain samples from the pons of PSP patients and age-matched controls, we found elevated levels of tau oligomers in PSP brains compared to controls, as well as increased total tau and phosphorylated tau in the form of NFTs. Tau oligomers were found in regions exhibiting typical PSP histological hallmarks, such as globose-shaped NFTs. The results reported here indicate that tau oligomers are important components of PSP pathology, along with NFTs. Studies supporting oligomers as the most toxic form of tau are becoming increasingly prevalent. While NFTs have been shown to correlate with disease progression in neurodegenerative tauopathies, such as AD, neuronal death and dysfunction begins to occur before the appearance of these large tau deposits [32-35]. The presence of tau annular protofibrils in glial cells in PSP as well as neurons suggests that tau oligomers may play a role in tau pathology in multiple cell types [36]. Tau oligomers lead to toxicity and cognitive deficits in mice [15] and are found to be elevated in the brains of $\mathrm{AD}$ patients $[16,19,21]$. However, this is the first time tau oligomers have been characterized in PSP.

Hyperphosphorylated NFTs have been known to be a main component of PSP and here we repeat results that PSP patients have increased levels of phosphorylated tau aggregates. Additionally, phosphorylated tau oligomers were detected, as well as non-phosphorylated, demonstrating the involvement of oligomeric species in the development of characteristic PSP pathology. Phosphorylation 


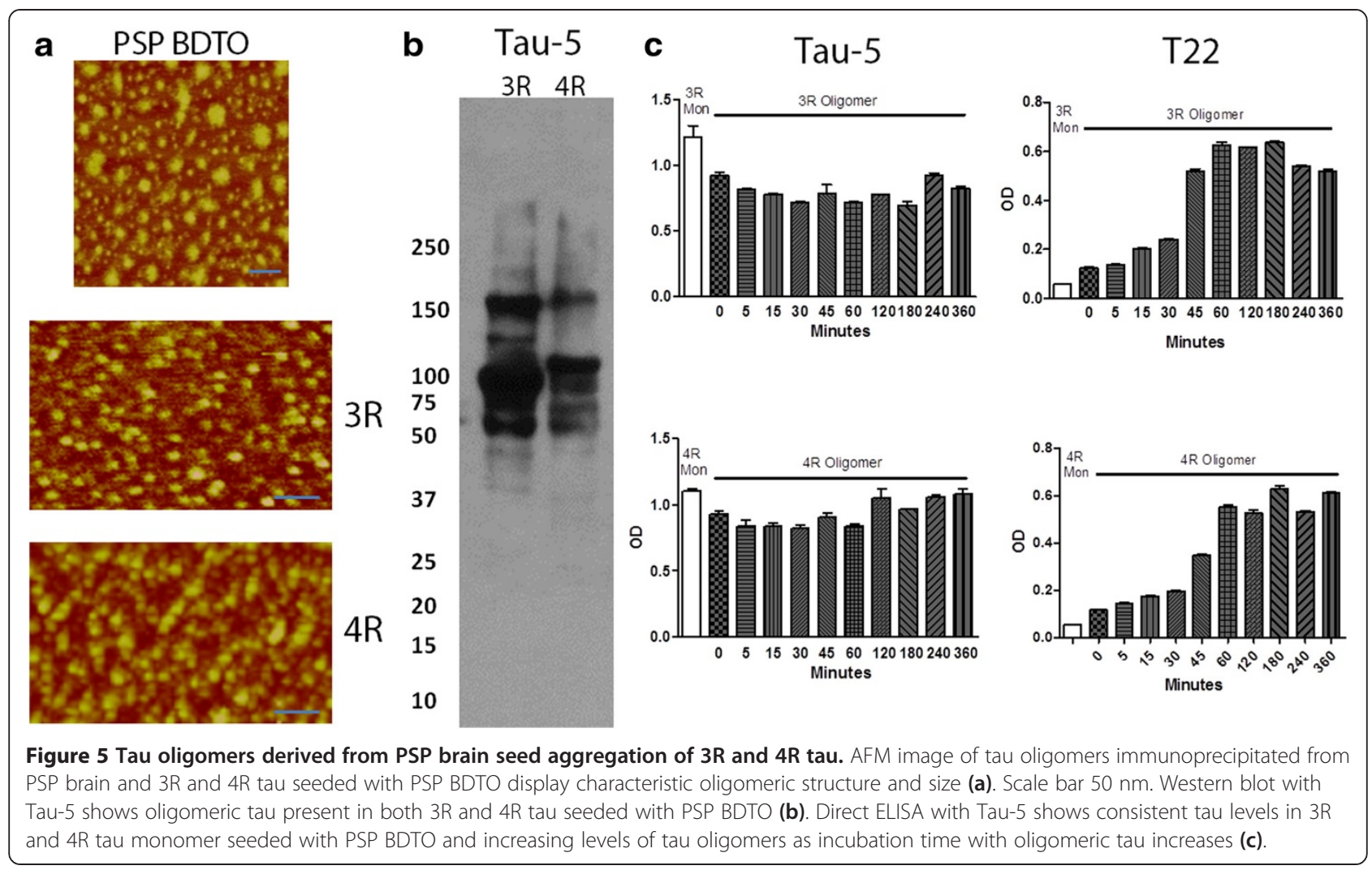

likely has important implications for toxicity, though the exact relationship between tau and phosphorylation state is not entirely certain. For one, evidence suggests that aberrant phosphorylation may increase the tendency of tau to form aggregates and that tau kinases are involved in the progression of tauopathies [37-39]. Phosphorylation state also regulates cellular localization of tau, with abnormal phosphorylation leading to the release of tau from the microtubules and redistribution from the axon to the somatodendritic compartment [40]. However, other studies have highlighted the relevance of dephosphorylated tau to toxicity and have suggested an increased ability for extracellular propagation $[41,42]$, implying that unphosphorylated tau oligomers detected in PSP brains may be equally important to disease progression.

The seeding of PSP brain-derived tau oligomers with both $3 \mathrm{R}$ and $4 \mathrm{R}$ tau induced the assembly of both tau isoforms into oligomers. Therefore, the mechanism by which tau pathology is induced in only $4 R$ tau in PSP remains unknown and may not depend upon differences in tau seeding. The ability of oligomeric tau derived from PSP to seed different types of tau may underlie the frequent co-existence of other neurodegenerative tauopathies in patients with PSP [43].

There are both overlaps and differences between all of the neurodegenerative diseases, hence more research is needed to understand the exact species of tau and post- translational modifications responsible for toxicity in distinct disorders, as well as to test whether the most toxic forms of tau are also those which are responsible for the spread of disease. In spite of differences in the ratio of tau isoforms, the histological hallmarks, and the localization of tau pathology in PSP, the results reported here suggest that tau oligomers, which are both phosphorylated and unphosphorylated, are involved in PSP, similarly to AD. This implies that there is likely a common mechanism for tauopathies, similarly to other wellcharacterized amyloids, such as amyloid beta, whereby oligomeric species underlie toxic effects. The ability of BDTO from PSP to seed the aggregation of $3 R$ and $4 R$ tau monomer supports other evidence for a prion-like mechanism for the spread of tau in neurodegenerative disease [18]. These results combined with recent studies showing the efficacy of passive immunotherapy targeting tau oligomers $[44,45]$ support the possibility of utilizing this strategy for therapeutics in PSP, as well as in other neurodegenerative tauopathies.

\section{Conclusions}

The results reported here show that tau oligomers are an important component of PSP pathology, similarly to what has been seen in other tauopathies, such as Alzheimer's disease. This suggests a common mechanism for tau toxicity in pure and mixed amyloid tauopathies. Tau 
oligomers derived from PSP human brain tissue were capable of seeding the oligomerization of both $3 \mathrm{R}$ and $4 R$ tau. As tau toxicity in PSP is primarily seen in $4 R$ tau, rather than $3 \mathrm{R}$ tau, this suggests that the selection mechanism for tau toxicity may be separate from seeding. The ability of PSP-derived oligomers to seed both forms of tau also provides a potential explanation for the high prevalence of co-morbidity of additional tauopathies in patients with PSP. These results support the use of tau oligomer-directed therapeutics for the prevention of disease progression in PSP and other tauopathies.

\section{Competing interests}

The authors declare that they have no competing interests.

\section{Authors' contributions}

RK, JEG, CLR and JT designed the study. JEG completed immunohistochemistry, characterization of tau oligomers and statistical analysis. US carried out immunoassays and biochemical analysis. CLR participated in the immunohistochemistry and analysis. MGM prepared protein samples and antibodies. JT provided human brain tissue and data interpretation. JEG and RK wrote the manuscript. All authors read and approved the final manuscript.

\section{Acknowledgments}

This work was supported by The Mitchell Center for Neurodegenerative Diseases and the Cullen Family Trust for Health Care.

\section{Author details}

${ }^{1}$ Mitchell Center for Neurodegenerative Diseases, University of Texas Medical Branch, Galveston, TX 77555, USA. ${ }^{2}$ Departments of Neurology, Neuroscience and Cell Biology, University of Texas Medical Branch, Galveston, TX 77555, USA. ${ }^{3}$ Department of Neuropathology, Johns Hopkins University, Baltimore, MD 21205, USA.

Received: 31 March 2014 Accepted: 10 June 2014

Published: 14 June 2014

\section{References}

1. Steele JC, Richardson JC, Olszewski J: Progressive supranuclear palsy. Arch Neurol 1964, 10:333-359.

2. Nath U, Ben-Shlomo Y, Thomson RG, Morris HR, Wood NW, Lees AJ, Burn DJ: The prevalence of progressive supranuclear palsy (Steele-RichardsonOlszewski syndrome) in the UK. Brain 2001, 124:1438-1449.

3. Goedert M, Spillantini MG, Jakes R, Rutherford D, Crowther RA: Multiple isoforms of human microtubule-associated protein tau: sequences and localization in neurofibrillary tangles of Alzheimer's disease. Neuron 1989, 3:519-526.

4. Panda D, Goode BL, Feinstein SC, Wilson L: Kinetic stabilization of microtubule dynamics. Biochemistry 1995, 34:11117-111127.

5. Ingelsson M, Ramasamy K, Russ C, Freeman SH, Orne J, Raju S, Matsui T, Growdon JH, Frosch MP, Ghetti B, Brown RH, Irizarry MC, Hyman BT: Increase in the relative expression of tau with four microtubule binding repeat regions in frontotemporal lobar degeneration and progressive supranuclear palsy brains. Acta Neuropathol 2007, 114:471-479.

6. Sergeant N, Wattez A, Delacourte A: Neurofibrillary degeneration in progressive supranuclear palsy and corticobasal degeneration. J Neurochem 1999, 72:1243-1249.

7. Arai T, Ikeda K, Akiyama H, Tsuchiya K, Yagishita S, Takamatsu J: Intracellular processing of aggregated tau differs between corticobasal degeneration and progressive supranuclear palsy. Clin Med 2001, 12:935-938.

8. Arai T, Ikeda K, Akiyama H, Nonaka T, Hasegawa M, Ishiguro K, Iritani S, Tsuchiya K, Iseki E, Yagishita S, Oda T, Mochizuki A: Identification of amino-terminally cleaved tau fragments that distinguish progressive supranuclear palsy from corticobasal degeneration. Ann Neurol 2004, 55:72-79.

9. Delacourte A, Sergeant N, Wattez A, Gauvreau D, Robitaille Y: Vulnerable neuronal subsets in Alzheimer's and Pick's disease are distinguished by their $\mathrm{T}$ isoform distribution and phosphorylation. Ann Neurol 1998, 43:193-204.
10. Arai T, Ikeda K, Akiyama H, Tsuchiya K, Iritani S, Ishiguro K, Yagishita S, Oda T, Odawara T, Iseki E: Different immunoreactivities of the microtubule-binding region of tau and its molecular basis in brains from patients with Alzheimer's disease, Pick's disease, progressive supranuclear palsy and corticobasal degeneration. Acta Neuropathol 2003, 105:489-498.

11. Yamada T, McGeer PL, McGeer EG: Appearance of paired nucleated, tau-positive glia in patients with progressive supranuclear palsy brain tissue. Neurosci Lett 1992, 135:99-102

12. Hauw JJ, Verny $M$, Delaère $P$, Cervera $P$, He $Y$, Duyckaerts C: Constant neurofibrillary changes in the neocortex in progressive supranuclear palsy. Basic differences with Alzheimer's disease and aging. Neurosci Lett 1990, 119:182-186.

13. Arima K: Ultrastructural characteristics of tau filaments in tauopathies: immuno-electron microscopic demonstration of tau filaments in tauopathies. Neuropathology 2006, 26:475-483.

14. Hof PR, Delacourte A, Bouras C: Distribution of cortical neurofibrillary tangles in progressive supranuclear palsy: a quantitative analysis of six cases. Acta Neuropathol 1992, 84:45-51.

15. Lasagna-Reeves CA, Castillo-Carranza DL, Sengupta U, Clos AL, Jackson GR, Kayed R: Tau oligomers impair memory and induce synaptic and mitochondrial dysfunction in wild-type mice. Mol Neurodegener 2011, 6:1-14.

16. Lasagna-Reeves CA, Castillo-Carranza DL, Sengupta U, Sarmiento J, Troncoso J, Jackson GR, Kayed R: Identification of oligomers at early stages of tau aggregation in Alzheimer's disease. FASEB J 2012, 26:1946-1959.

17. Lasagna-Reeves CA, Castillo-Carranza DL, Sengupta U, Guerrero-Munoz MJ, Kiritoshi T, Neugebauer V, Jackson GR, Kayed R: Alzheimer brain-derived tau oligomers propagate pathology from endogenous tau. Sci Rep 2012, 2:700.

18. Gerson JE, Kayed R: Formation and propagation of tau oligomeric seeds. Front Neurol 2013, 4. doi:10.3389/fneur.2013.00093.

19. Maeda S, Sahara N, Saito Y, Murayama S, Ikai A, Takashima A: Increased levels of granular tau oligomers: an early sign of brain aging and Alzheimer's disease. Neurosci Res 2006, 54:197-201.

20. Maeda S, Sahara N, Saito Y, Murayama M, Yoshiike Y, Kim H, Miyasaka T, Murayama S, Ikai A, Takashima A: Granular tau oligomers as intermediates of tau filaments. Biochemistry 2007, 46:3856-3861.

21. Patterson KR, Remmers C, Fu Y, Brooker S, Kanaan NM, Vana L, Ward S, Reyes JF, Philibert K, Glucksman MJ, Binder LI: Characterization of prefibrillar tau oligomers in vitro and in Alzheimers disease. $J$ Biol Chem 2011, 286:23063-23076.

22. Tai H-C, Serrano-Pozo A, Hashimoto T, Frosch MP, Spires-Jones TL, Hyman BT: The synaptic accumulation of hyperphosphorylated tau oligomers in Alzheimer disease is associated with dysfunction of the ubiquitinproteasome system. Am J Pathol 2012, 181:1426-1435.

23. Kopeikina KJ, Carlson GA, Pitstick R, Ludvigson AE, Peters A, Luebke I, Koffie RM, Frosch MP, Hyman BT, Spires-Jones TL: Tau accumulation causes mitochondrial distribution deficits in neurons in a mouse model of tauopathy and in human Alzheimer's disease brain. Am J Pathol 2011, 179:2071-2082.

24. Berger Z, Roder H, Hanna A, Carlson A, Rangachari V, Yue M, Wszolek Z, Ashe K, Knight J, Dickson D, Andorfer C, Rosenberry TL, Lewis J, Hutton M, Janus C: Accumulation of pathological tau species and memory loss in a conditional model of tauopathy. J Neurosci 2007, 27:3650-3662.

25. Polydoro M, Acker CM, Duff K, Castillo PE, Davies P: Age-dependent impairment of cognitive and synaptic function in the htau mouse model of tau pathology. J Neurosci 2009, 29:10741-10749.

26. SantaCruz K, Lewis J, Spires T, Paulson J, Kotilinek L, Ingelsson M, Guimaraes A, DeTure M, Ramsden M, McGowan E, Forster C, Yue M, Orne J, Janus C, Mariash A, Kuskowski M, Hyman B, Hutton M, Ashe KH: Tau suppression in a neurodegenerative mouse model improves memory function. Science 2005, 309:476-481.

27. Yoshiyama Y, Higuchi M, Zhang B, Huang SM, Iwata N, Saido TC, Maeda J, Suhara T, Trojanowski JQ, Lee VM: Synapse loss and microglial activation precede tangles in a P301S tauopathy mouse model. Neuron 2007, 53:337-351.

28. Sahara N, DeTure M, Ren Y, Ebrahim AS, Kang D, Knight J, Volbracht C, Pedersen JT, Dickson DW, Yen SH, Lewis J: Characteristics of TBSextractable hyperphosphorylated tau species: aggregation intermediates in rTg4510 mouse brain. J Alzheimers Dis 2013, 33:249-263.

29. Golbe LI: The tau of PSP: a long road to treatment. Mov Disord 2014, 29:431-434 
30. Lasagna-Reeves CA, Glabe CG, Kayed R: Amyloid- $\beta$ annular protofibrils evade fibrillar fate in Alzheimer disease brain. J Biol Chem 2011, 286:22122-22130.

31. Lasagna-Reeves CA, Castillo-Carranza DL, Guerrero-Muñoz MJ, Jackson GR, Kayed R: Preparation and characterization of neurotoxic tau oligomers. Biochemistry 2010, 49:10039-10041.

32. Gomez-Isla T, Hollister R, West H, Mui S, Growdon JH, Petersen RC, Parisi JE, Hyman BT: Neuronal loss correlates with but exceeds neurofibrillary tangles in Alzheimer's disease. Ann Neurol 1997, 41:17-24.

33. Vogt BA, Vogt LJ, Vrana KE, Gioia L, Meadows RS, Challa VR, Hof PR, Van Hoesen GW: Multivariate analysis of laminar patterns of neurodegeneration in posterior cingulate cortex in Alzheimer's disease. Exp Neurol 1998, 153:8-22.

34. Terry RD: Do neuronal inclusions kill the cell? J Neural Transm Suppl 2000, 59:91-93.

35. van de Nes JA, Nafe R, Schlote W: Non-tau based neuronal degeneration in Alzheimer's disease - an immunocytochemical and quantitative study in the supragranular layers of the middle temporal neocortex. Brain Res 2008, 1213:152-165.

36. Lasagna-Reeves C, Sengupta U, Castillo-Carranza D, Gerson J, GuerreroMunoz M, Troncoso J, Jackson G, Kayed R: The formation of tau pore-like structures is prevalent and cell specific: possible implications for the disease phenotypes. Acta Neuropathol Commun 2014, 2:56.

37. Alonso ADC, Zaidi T, Novak M, Barra HS, Grundke-lqbal I, lqbal K: Interaction of tau isoforms with Alzheimer's disease abnormally hyperphosphorylated tau and in vitro phosphorylation into the disease-like protein. J Biol Chem 2001, 276:37967-37973.

38. Alonso ADC, Zaidi T, Novak M, Grundke-lqbal I, Iqbal K: Hyperphosphorylation induces self-assembly of $\tau$ into tangles of paired helical filaments/straight filaments. PNAS 2001, 98:6923-6928.

39. Pérez M, Cuadros R, Smith MA, Perry G, Avila J: Phosphorylated, but not native, tau protein assembles following reaction with the lipid peroxidation product, 4-hydroxy-2-nonenal. FEBS Lett 2000, 486:270-274.

40. Greenwood JA, Johnson GWW: Localization and in situ phosphorylation state of nuclear tau. Exp Cell Res 1995, 220:332-337.

41. Díaz-Hernández M, Gómez-Ramos A, Rubio A, Gómez-Villafuertes R, Naranjo $J R$, Miras-Portugal MT, Avila J: Tissue-nonspecific alkaline phosphatase promotes the neurotoxicity effect of extracellular tau. J Biol Chem 2010, 285:32539-32548.

42. Pooler AM, Phillips EC, Lau DHW, Noble W, Hanger DP: Physiological release of endogenous tau is stimulated by neuronal activity. EMBO Rep 2013, 14:389-394.

43. Keith-Rokosh J, Ang LC: Progressive supranuclear palsy: a review of co-existing neurodegeneration. Can J Neurol Sci 2008, 35:602-608.

44. Castillo-Carranza DL, Gerson JE, Sengupta U, Guerrero-Muñoz MJ, LasagnaReeves CA, Kayed R: Specific targeting of tau oligomers in htau mice prevents cognitive impairment and tau toxicity following injection with brain-derived tau oligomeric seeds. J Alzheimers Dis, 40:S97-S111.

45. Castillo-Carranza DL, Sengupta U, Guerrero-Muñoz MJ, Lasagna-Reeves CA Gerson JE, Singh G, Estes DM, Barrett ADT, Dineley KT, Jackson GR, Kayed R: Passive immunization with tau oligomer monoclonal antibody reverses tauopathy phenotypes without affecting hyperphosphorylated neurofibrillary tangles. J Neurosci 2014, 34:4260-4272.

doi:10.1186/2051-5960-2-73

Cite this article as: Gerson et al:: Characterization of tau oligomeric seeds in progressive supranuclear palsy. Acta Neuropathologica Communications 2014 2:73.

\section{Submit your next manuscript to BioMed Central and take full advantage of:}

- Convenient online submission

- Thorough peer review

- No space constraints or color figure charges

- Immediate publication on acceptance

- Inclusion in PubMed, CAS, Scopus and Google Scholar

- Research which is freely available for redistribution

Submit your manuscript at www.biomedcentral.com/submit
Ciomed Central 